\title{
Graded multi-label classification: compromise between handling label relations and limiting error propagation
}

\author{
Khalil Laghmari \\ Laboratoire Informatique de Mohammedia, \\ FSTM, Hassan II University of Casablanca, \\ BP 146 Mohammedia 20650 Maroc. \\ Sorbonne Universités, \\ UPMC Univ Paris 06, \\ CNRS, LIP6 UMR 7606, \\ 4 place Jussieu 75005 Paris, France. \\ laghmari.khalil@gmail.com
}

\author{
Christophe Marsala \\ Sorbonne Universités, \\ UPMC Univ Paris 06, \\ CNRS, LIP6 UMR 7606, \\ 4 place Jussieu 75005 Paris, France \\ christophe.marsala@lip6.fr
}

\author{
Mohammed Ramdani \\ Laboratoire Informatique de Mohammedia, \\ FSTM, Hassan II University of Casablanca, \\ BP 146 Mohammedia 20650 Maroc. \\ ramdani@ fstm.ac.ma
}

\begin{abstract}
In graded multi-label classification (GMLC), each data can be assigned to multiple labels according to a degree of membership on an ordinal scale, and with respect to label relations. For example, in a movie catalog web page, a five stars action movie should be at least a one star suspense movie. Ignoring those relations can lead to inconsistent predictions, but if they are considered, then a prediction error for one label will be propagated to all related labels. Most of existing approaches either ignore label relations, or can learn only relations fitting a predefined imposed structure. This paper is motivated by the lack of a study analysing the compromise between handling label relations and limiting error propagation in GMLC, and by the fact that there is no known approach giving a control on that compromise to allow such a study. In this paper, a new meta-classifier with two main advantages is proposed for GMLC. Firstly, no predefined structure is imposed for learning label relations, and secondly, the meta-classifier is based on three measures giving control on the studied compromise. The studied compromise is analysed according to its impact on the classifier complexity and on hamming-loss evaluation measure. A comparison to three existing approaches shows that the proposed meta-classifier is competitive according to hammingloss evaluation measure, and it is the most stable classifier according to hamming-loss standard deviation.
\end{abstract}

\section{INTRODUCTION}

Graded multi-label classification (GMLC) is the task of assigning one or more labels to each data according to an ordinal scale of membership degrees $M$. It was recently introduced [1] as a generalization of the multi-label classification task (MLC) [2].

The most known source for GMLC data is catalogue web pages, where movies or animes are assigned to different categories such as action, suspense, and humour using a one-to-five star rating. Data involving the task of GMLC can be found in many other domains such as chemistry, where molecules have multiple odours with different intensities ranging from very weak to very strong.
Relations can exist between labels according to $M$. For example, high ranked action movies should contain at least little suspense, and molecules having a very strong intensity of jasmine odour can not have the smell of musk simultaneously.

Those relations, if well learned, are supposed to give a better understanding of hidden knowledge in data, but actually they lead to a serious dilemma:

- On the one hand, considering label relations has the advantage of making consistent predictions, but it has the disadvantage of allowing error propagation because a prediction error for one label will be propagated to all related labels.

- On the other hand, learning a classifier while ignoring those relations has the advantage of making independent predictions which prevents error propagation, but it has the disadvantage of making inconsistent predictions since label relations are ignored.

Label relation dilemma is an inherent challenge in all MLC tasks and not only specific to GMLC. However, this challenge has not received enough attention in the literature: first MLC approaches assume label independence, and later approaches focused only on how to handle label relations.

Learning all label relations can lead to cyclic dependencies between labels. Most of existing MLC approaches avoid this problem by setting a non cyclic dependence structure, and then try to learn only relations fitting the predefined structure.

Another limitation of existing MLC approaches is that they can handle only co-occurrence relations, while GMLC data can encapsulate also order relations based on the ordinal scale of membership degrees.

To overcome the limitations of existing MLC approaches, and to answer the challenge of label relation dilemma in GMLC, we propose a new meta-classifier named PSI-MC with two main advantages: Firstly, it allows label relation learning from GMLC data without imposing a predefined 
structure, and secondly, based on three measures named preselection, selection, and interest measures, it allows controlling the compromise between learning label relations and limiting error propagation.

The paper is organized as follows: in Section II we review the most used MLC approaches, in Section III we review some well-known GMLC approaches, and in Section IV we describe the proposed meta-classifier PSI-MC. Experiments on real datasets are discussed in Section V. Conclusions and ideas for future works are presented in Section VI.

\section{Multi-LABEL CLASSIFICATION}

Let $C=\left\{c_{l}\right\}_{1 \leq l \leq k}$ be the set of labels, and $X=\left\{x_{i}\right\}_{1 \leq i \leq n}$ be the set of data. Each data $x_{i}$ is a vector $\left(x_{i j}\right)_{1 \leq j \leq p}$ where $x_{i j}$ is the value corresponding to the $j^{t h}$ attribute. $y_{i} \subseteq C$ is the set of labels associated to $x_{i}$. The MLC task is to learn a classifier $H: X \rightarrow \mathcal{P}(C)$ mapping each data to the correct set of associated labels. The MLC task is answered either by extending a mono-label classifier, or by transforming multilabel data to mono-label data.

Many mono-label classifiers were adapted to handle multilabel data, such as the K-Nearest Neighbours algorithm [3] [4], the naive bayes algorithm [5], the Support Vector machines [6] [7], the decision trees [8] [9], and the neural networks [10] [11]. In this paper we are more interested in transformation methods [12] because they can be used with any mono-label classifier.

There are three families of learning approaches based on transformation methods: Learning one multi-classes classifier, learning a binary classifier for each label, and learning a binary classifier for each two different labels.

\section{A. Learning one multi-classes classifier}

Label power set (LP) is a straightforward approach considering each label set as a single distinct label. One disadvantage of this approach is the class imbalance problem encountered when some label sets are not frequent. The idea behind pruned problem transformation approach (PPT) [13] is to remove infrequent label sets in order to overcome the class imbalance problem, but this leads to an information loss due to label truncation. A pruned problem transformation with no information loss (PPT-n) [13] is an improvement of PPT. Instead of removing infrequent label sets, PPT-n divide them to frequent subsets of labels. A common disadvantage between LP, PPT, and PPT-n approaches is that they can not predict an unseen label set in the training set. This limitation is overcome by the PPT-ext approach [13].

\section{B. Learning a binary classifier for each label}

In binary relevance approach (BR), a binary classifier $H_{l}$ : $X \rightarrow\{0,1\}$ is learned for each label $c_{l} \in C$. The labels relevant to predict are given by $H(x)=\left\{c_{l}, H_{l}(x)=1\right\}_{1 \leq l \leq k}$. This approach learns independent classifiers and therefore can not learn label relations. Classifier chains approach (CC) [14] overcome this limitation using a chained structure depending on a predefined order for labels. Each classifier $H_{l}$ is allowed to use the prediction result of all previous classifiers
$\left\{H_{l^{\prime}}\right\}_{1<l^{\prime}<l}$ in order to make its own prediction. In classifier treillis approach (CT) [15] a directed graph is built instead of a chained structure. Label correlation is computed between each pair of labels, then classifiers are placed on nodes according to label correlation. Each classifier is allowed to use the prediction result of all classifiers corresponding to parent nodes.

\section{Learning a binary classifier for each two different labels}

In ranking by pairwise comparison approach (RPC) [16], a binary classifier $H_{l l^{\prime}}$ is learned for each label pair $c_{l} \neq c_{l^{\prime}}$. Each $H_{l l^{\prime}}$ is used to predict whether a data is associated to the label $c_{l}$ or $c_{l^{\prime}}$. A label ranking is outputted using a majority vote aggregation but with no separation between relevant and irrelevant labels. This problem is solved by calibrated label ranking approach (CLR) [17]. The idea is to train $k$ more classifiers using an additional label $c_{0}$. For each classifier $H_{l 0}, l \in[1, k]$, data not associated with the label $c_{l}$ is considered as associated with the additional label $c_{0}$. Labels predicted more times then $c_{0}$ are relevant labels, and the remaining are considered as irrelevant labels.

\section{GRADED MULTI-LABEL CLASSIFICATION}

\section{A. GMLC and fuzzy sets}

GMLC data can be viewed as fuzzy sets [18]. The only difference is that in fuzzy sets a numeric scale is used for membership degrees $M=[0,1]$, while in GMLC an ordinal scale is used $M=\left\{m_{1}<\ldots<m_{s}\right\}$. The interest of GMLC is related to data acquisition because it is easier for annotators to give their opinions on an ordinal scale. Each data $x_{i}$ is associated with a fuzzy set $y_{i}$ where $\lambda_{i}: C \rightarrow M$ is the mapping function of $x_{i}$ associating each label $c_{l} \in C$ to its membership grade.

A fuzzy set can be described by the vertical representation using the membership function, or by the horizontal representation using $\alpha$-cuts. In a similar way, the GMLC task can be solved using either a vertical or an horizontal decomposition, or both [1].

\section{B. Decomposing the GMLC problem}

Using the vertical decomposition, $k$ classifiers are trained, one for each label $c_{l} \in C$, so that each classifier $H_{l}: X \rightarrow M$ predicts the membership grade for the label $c_{l}$.

Using the horizontal decomposition, $s-1$ classifiers are trained, one for each grade $m_{g} \in\left\{m_{2}, \ldots, m_{s}\right\}$, so that each classifier $H_{g}: X \rightarrow \mathcal{P}(C)$ predicts the set of associated labels according to a membership grade at least equals to $m_{g}$. There is no need to train a classifier for $m_{1}$ because the corresponding label set is $H_{1}(x)=C$.

If a label $c_{l}$ is predicted by a classifier $H_{g}$, it is expected from all classifiers $\left\{H_{g^{\prime}}\right\}_{1 \leq g^{\prime} \leq g}$ to predict $c_{l}$ as well. In regard to this hierarchical property, the trained classifiers are not completely independent, hence the horizontal decomposition in theory, can learn better label relations than the vertical decomposition. However, it is not guaranteed that the hierarchical property is satisfied by trained classifiers. An aggregation function is generally used to handle this case. 
Combining both decompositions can be done starting first by the vertical decomposition, then applying an horizontal decomposition, or the opposite. Indeed, the task of each vertical classifier is an ordinal classification [19] [20] and can be solved horizontally using $s-1$ binary classifiers, and the task of each horizontal classifier can be solved vertically using the BR approach.

\section{Solving the GMLC problem using a pairwise approach}

The task of GMLC can be solved also using a pairwise approach like CLR. The three methods named Horizontal CLR, Full CLR, and Joined CLR are all based on the idea of using multiple calibration labels [21]. The key idea is to use $s-1$ virtual labels: $V=\left\{v_{g}\right\}_{1 \leq g \leq s-1}$ with fixed membership grades: $\forall i \in[1, n]: m_{g}<\lambda_{i}\left(v_{g}\right)<m_{g+1}$. $v_{g}$ is used to denote (for simplicity) both the virtual label and its corresponding membership grade: $\lambda_{i}\left(v_{g}\right)=v_{g}$.

For the Horizontal CLR approach, an horizontal decomposition is first performed, then each MLC task $g$ from the obtained $s-1$ tasks is solved using CLR, with $v_{g}$ as the cutting point between relevant and irrelevant labels.

For the Full CLR approach, a classifier is trained for each label pair in $C \cup V$. All virtual labels are considered as cutting points. The membership grade $m_{g}$ is predicted for a label $c_{l} \in C$ if $m_{g}$ is the highest membership grade for which $c_{l}$ is predicted more times than $v_{g}$.

One drawback of Full CLR approach, is that for a classifier $H_{l l^{\prime}}$, a data $x_{i}$ is considered positive if $\lambda_{i}\left(c_{l}\right)>\lambda_{i}\left(c_{l^{\prime}}\right)$, and negative otherwise, regardless of the difference between membership grades.

Joined CLR answers this problem by combining both Horizontal CLR and Full CLR approaches. Indeed, each MLC task for the Horizontal CLR approach is solved using all virtual labels as cutting points, instead of using only one cutting point.

The three CLR based approaches discussed in this section are also used in experiment section (V), where our proposed meta-classifier is compared to them.

\section{A NEW META-CLASSIFIER FOR GRADED MULTI-LABEL CLASSIFICATION}

\section{A. Key ideas}

The first key idea of the proposed meta-classifier is to learn an initial set of $k$ multi-class classifiers $H^{0}=\left\{H_{l}\right\}_{1 \leq l \leq k}$, one for each label, where the training set for a classifier $H_{l}$ includes membership grades of labels $\left\{c_{l^{\prime}}\right\}_{l^{\prime} \neq l}$ as descriptive numeric attributes, which means that the prediction by a classifier $H_{l}$ can depend on the prediction result of other classifiers $\left\{H_{l^{\prime}}\right\}_{l^{\prime} \neq l}$. This allows learning label relations considering membership grades without fixing a predefined structure, but it does not prevent learning cyclic dependencies.

The second key idea is to avoid cyclic dependencies by replacing involved classifiers. The compromise between handling label relations and limiting error propagation is defined by the way we select which classifier to replace first, and by the way we select alternative classifiers that the new classifier can depend on. The final set of classifiers without cyclic dependencies is the one used to make predictions $\mathbb{H}=\left\{\mathbb{H}_{L}\right\}_{1<L<k}$.

Initially, the final classifier set is empty: $\mathbb{H} \leftarrow \emptyset$, and the set of classifiers not yet added to $\mathbb{H}$ is $H^{0}-\mathbb{H}=H$. Our proposed meta-classifier PSI-MC removes iteratively classifiers from $H$, and adds them directly or after being replaced to $\mathbb{H}$, until $|H|=0$ and consequently $|\mathbb{H}|=k$.

\section{B. Measures}

A pre-selection measure $\mathbb{P}: H \rightarrow\{0,1\}$

is used to fill the set of candidate classifiers for replacement: $\left\{H_{l} \in H, \mathbb{P}\left(H_{l}\right)=1\right\}_{1 \leq l \leq k}$. Note that our objective is not only to solve cyclic dependencies but also to answer the challenge of label relation dilemma. Hence, in order to reduce the effect of error propagation, even if a classifier is not involved in a cyclic dependency, it can be a candidate for replacement if it depends on too many other classifiers.

Classifiers not candidates for replacement are added directly to the final classifier set:

$\mathbb{H} \leftarrow \mathbb{H} \cup\left\{H_{l} \in H, \mathbb{P}\left(H_{l}\right)=0\right\}_{1 \leq l \leq k}$.

A selection measure $\mathbb{S}: \mathcal{P}(H) \rightarrow H$ is used to select one classifier to be replaced from the set of candidate classifiers. For example, it can be either the classifier that depends on the lowest number of classifiers to reduce information loss, or the classifier that depends on the highest number of classifiers to reduce error propagation. It could also be the classifier whose the highest number of classifiers depend on to resolve more cyclic dependencies.

A measure of chaining interest $\mathbb{I}: \mathbb{H} \rightarrow\{0,1\}$ is used to decide whether the new classifier to build can depend on a final classifier $\mathbb{H}_{L}: \mathbb{I}\left(\mathbb{H}_{L}\right)=1$ or not: $\mathbb{I}\left(\mathbb{H}_{L}\right)=0$.

pre-selection, selection, and chaining interest measures are called the PSI-measures. In the following, we investigate their impact on synthetic data before analysing experiment results on real datasets.

\section{Analysing the impact of PSI-measures using synthetic data}

In TABLE I, $X=\left\{x_{i}\right\}_{1<i<10}$ is the training set, $C=\left\{c_{l}\right\}_{1<l<5}$ is the label set, $M=\left\{m_{g}\right\}_{1<g<4}=$ $\{0,1,2,3\}$ is the set of membership grades, and $\left\{a_{1}, a_{2}\right\}$ is the set of descriptive attributes.

\begin{tabular}{|c|c|c||c|c|c|c|c|}
\hline & $a_{1}$ & $a_{2}$ & $c_{1}$ & $c_{2}$ & $c_{3}$ & $c_{4}$ & $c_{5}$ \\
\hline$x_{1}$ & 20 & 20 & 0 & 0 & 3 & 0 & 0 \\
$x_{2}$ & 30 & 40 & 1 & 0 & 3 & 0 & 0 \\
$x_{3}$ & 20 & 30 & 0 & 0 & 3 & 0 & 0 \\
$x_{4}$ & 20 & 10 & 0 & 0 & 0 & 0 & 3 \\
$x_{5}$ & 50 & 40 & 2 & 3 & 0 & 1 & 2 \\
$x_{6}$ & 50 & 20 & 2 & 3 & 0 & 1 & 2 \\
$x_{7}$ & 10 & 10 & 0 & 1 & 2 & 2 & 3 \\
$x_{8}$ & 10 & 30 & 0 & 3 & 1 & 2 & 2 \\
$x_{9}$ & 10 & 10 & 0 & 1 & 2 & 2 & 3 \\
$x_{10}$ & 10 & 50 & 0 & 3 & 1 & 2 & 2 \\
\hline \multicolumn{6}{c}{ TABLE I } \\
\multicolumn{6}{c}{ GMLC DATA }
\end{tabular}

In the following, we use decision trees as base classifiers because they are easily interpreted, however the proposed meta-classifier can be used with any other base classifier. 
Fig. 1 shows the obtained decision trees for $H^{0}$ using the weka implementation [22] of the $C 4.5$ algorithm [23], and Fig. 2 shows the corresponding dependency graph.

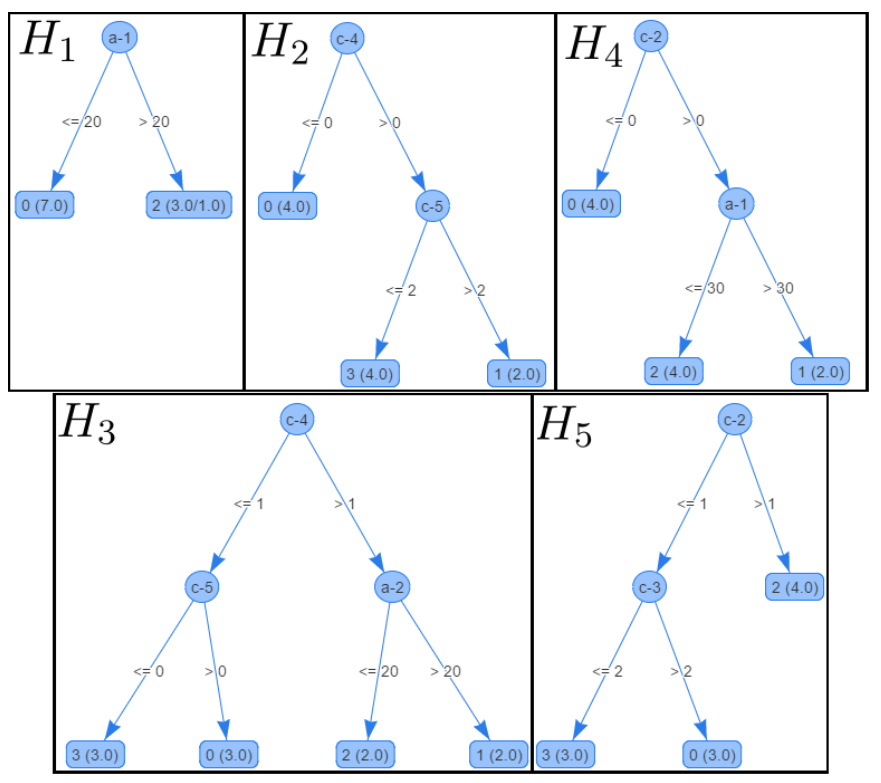

Fig. 1. Decision trees for $H^{0}$

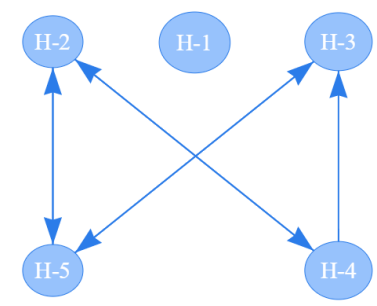

Fig. 2. Dependency graph for $H^{0}$

Let $D^{\rightarrow}: H \rightarrow \mathcal{P}(H)$ be the function giving for each classifier $H_{l}$ the set of classifiers depending on it, and $D^{\leftarrow}$ : $H \rightarrow \mathcal{P}(H)$ be the function giving for each classifier $H_{l}$ the set of classifiers that it depends on.

In this example, we choose to make all non independent classifiers candidates for replacement:

$\mathbb{P}\left(H_{l}\right)=0$ if $D^{\leftarrow}\left(H_{l}\right)=\emptyset, 1$ otherwise.

$H_{1}$ is independent, hence it is removed from $H$ and added directly to $\mathbb{H}$ (Fig. 3).
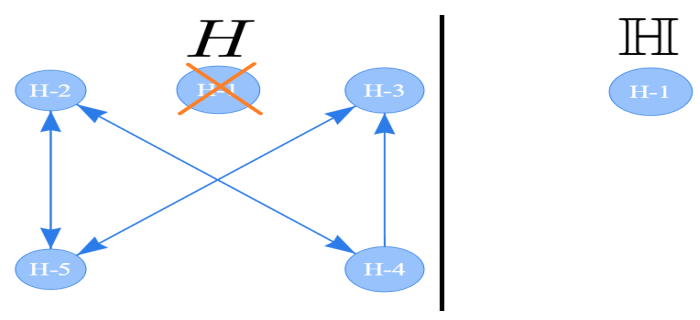

Fig. 3. Moving $H_{1}$ from $H$ to $\mathbb{H}$
The remaining classifiers are all dependent. One of them should be selected according to a selection measure. In this example we choose to select the classifier allowing us to solve the highest number of cyclic dependencies:

$\mathbb{S}(H)=\underset{H_{l} \in H}{\operatorname{argmax}}\left(\left|D^{\rightarrow}\left(H_{l}\right)\right|\right)$.

We have $\left|D^{\rightarrow}\left(H_{3}\right)\right|=1<\left|D^{\rightarrow}\left(H_{2}\right)\right|=\left|D^{\rightarrow}\left(H_{4}\right)\right|=$ $\left|D^{\rightarrow}\left(H_{5}\right)\right|=2$. The selection measure outputs the first classifier with the highest value which is $H_{2}$ in this case.

$H_{2}$ is to be replaced by another classifier $H_{2}^{\prime}$, and we have the choice to chain it with $\mathbb{H}_{1}$ or to make it independent. In this example the chaining interest measure is given by $\mathbb{I}\left(\mathbb{H}_{L}\right)=$ $0, \forall \mathbb{H}_{L} \in \mathbb{H}$.

Fig. 4 shows the updated dependency graphs for $H$ and $\mathbb{H}$ after replacing $H_{2}$ by $H_{2}^{\prime}$.

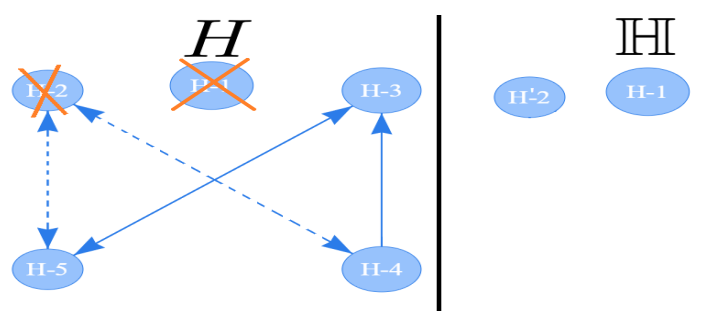

Fig. 4. Replacing $\mathrm{H}_{2}$ by $H_{2}^{\prime}$

Note that after removing $\mathrm{H}_{2}$ from $\mathrm{H}, \mathrm{H}_{4}$ becomes independent and then it is added directly to $\mathbb{H}$ (Fig. 5). Also note that since $H_{4}$ was depending on $H_{2}$ in $H$, it is now depending on $H_{2}^{\prime}$ in $\mathbb{H}$. This shows that the selection measure choosing which classifier to replace first, has also an impact on the learned label relations.

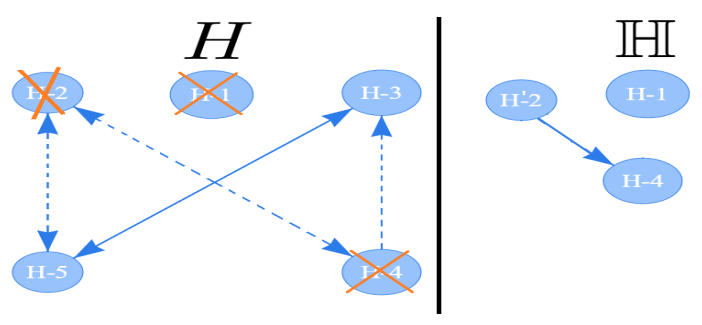

Fig. 5. Moving $H_{4}$ from $H$ to $\mathbb{H}$

We have $D^{\leftarrow}\left(H_{3}\right)=D^{\leftarrow}\left(H_{5}\right)=1$, hence $\mathbb{P}\left(H_{3}\right)=$ $\mathbb{P}\left(H_{5}\right)=1 . H_{3}$ and $H_{5}$ are both candidates for replacement, and since we have $\left|D^{\rightarrow}\left(H_{3}\right)\right|=\left|D^{\rightarrow}\left(H_{5}\right)\right|=1$, then $\mathbb{S}(H)=H_{3}$.

$H_{3}$ is replaced by an independent classifier $H_{3}^{\prime}$ according to the chaining interest measure $\mathbb{I}$ (Fig. 6).

After removing $\mathrm{H}_{3}$ from $H, H_{5}$ becomes independent and it is added directly to $\mathbb{H}$ (Fig. 7). Since $H_{5}$ was depending on both $\mathrm{H}_{2}$ and $\mathrm{H}_{3}$ in $\mathrm{H}$, it is now depending on $\mathrm{H}_{2}^{\prime}$ and $\mathrm{H}_{3}^{\prime}$ in $\mathbb{H}$.

Fig. 8 shows the dependency graph for $H^{0}$, compared to the one for $\mathbb{H}$ using a chaining interest measure always equals to 0 (the same used in the example), and to the one for $\mathbb{H}$ using a chaining interest measure always equals to 1 . Note 


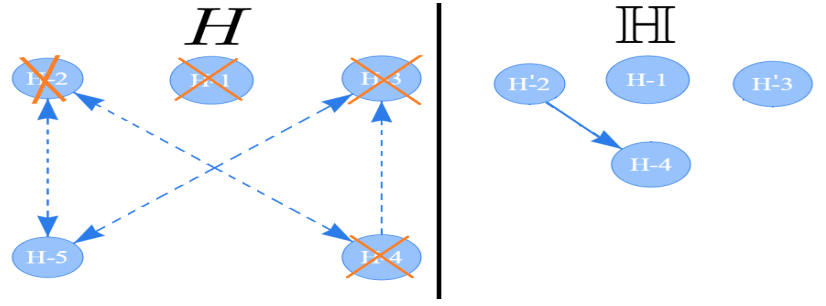

Fig. 6. Replacing $H_{3}$ by $H_{3}^{\prime}$

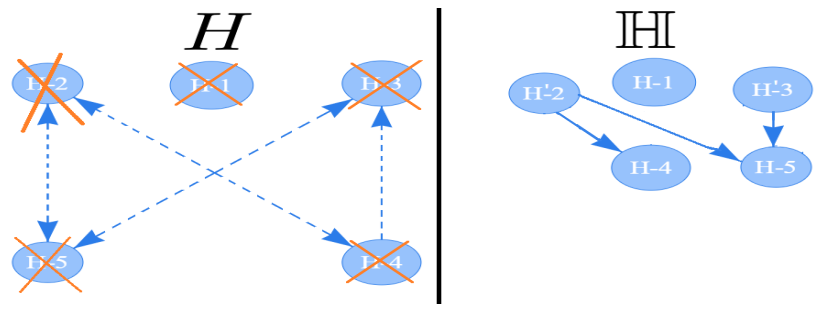

Fig. 7. Moving $H_{5}$ from $H$ to $\mathbb{H}$

that with $\mathbb{I}=1$ new label relations are learned instead of the initial cyclic relations.

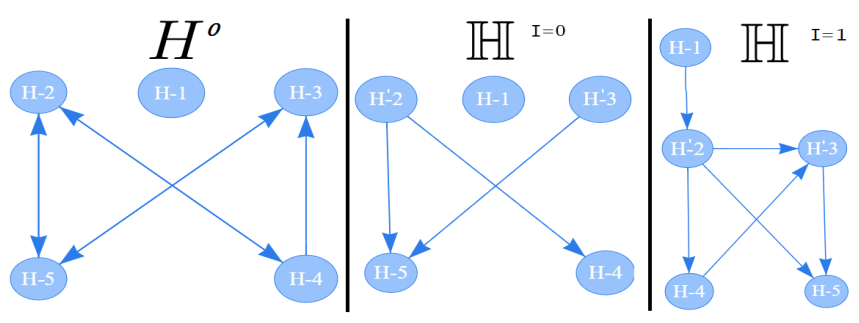

Fig. 8. Dependency graphs

In summary, by marking classifiers to be replaced, the preselection measure $\mathbb{P}$ can reduce learned label relations to minimize the risk of error propagation. The learned relation structure can change according to the order of replaced classifiers, which is determined by the selection measure $\mathbb{S}$. The chaining interest measure $\mathbb{I}$ controls allowed label relations: predictions are supposed to be more consistent by allowing replaced classifiers to learn many relations, and by doing the opposite the risk of error propagation is reduced.

\section{EXPERIMENTS}

\begin{tabular}{|c|c|c|c|c|}
\hline dataset & instances & attributes & labels & grades \\
\hline BelaE-5 & 1930 & 45 & 5 & $\{0,1,2,3,4\}$ \\
BelaE-10 & 1930 & 40 & 10 & $\{0,1,2,3,4\}$ \\
molecules & 2600 & 15 & 81 & $\{0,1,2,3,4,5,6\}$ \\
\hline \multicolumn{3}{c}{ TABLE II }
\end{tabular}

DESCRIPTION OF USED DATASETS

TABLE II describes datasets used in our experiments. The original BelaE data ${ }^{1}$ is collected from the answers of 1930 graduate students about the importance of 48 properties of their future jobs. Descriptive attributes are age and sex of students, and labels are the 48 properties. To overcome the

\footnotetext{
${ }^{1}$ http://www.ke.tu-darmstadt.de/resources/GMLC
}

problem of insufficient attributes, 50 datasets are randomly generated considering only $k$ labels as target labels and the remaining labels as descriptive attributes. In order to compare our proposed meta-classifier results to reported results of Horizontal CLR, Full CLR, and Joined CLR approaches [21], we used the same 50 datasets for $k=5$, the same 50 datasets for $k=10$, and the same base classifier (weka implementation of C4.5) as in [21]. Results averaged over 10 folds crossvalidation are shown in TABLE III, where hamming-loss is extended to the GMLC case as described in [21].

\begin{tabular}{r|r|c}
\hline \multicolumn{1}{c|}{ Dataset } & meta-classifier & hamming-loss average and standard deviation \\
\hline \multirow{4}{*}{ BelaE-5 } & Full CLR & $0.3397 \pm 5.79$ \\
& Joined CLR & $0.1796 \pm 1.31$ \\
& Horizontal CLR & $0.1577 \pm 1.53$ \\
& PSI-MC $(I=1)$ & $0.1891 \pm 0.0956$ \\
& PSI-MC $(I=0)$ & $0.1889 \pm 0.0949$ \\
\hline \multirow{5}{*}{ BelaE-10 } & Full CLR & $0.3544 \pm 3.70$ \\
& Joined CLR & $0.1792 \pm 0.87$ \\
& Horizontal CLR & $0.1513 \pm 0.95$ \\
& PSI-MC $(I=1)$ & $0.1894 \pm 0.0721$ \\
& PSI-MC $(I=0)$ & $0.1884 \pm 0.0709$ \\
\hline
\end{tabular}

TABLE III

EVALUATION OF GRADED MULTI-LABEL CLASSIFIERS USING BELAE DATASET

Our proposed meta-classifier is at least better than Full CLR according to hamming-loss (TABLE III), and almost as good as Joined CLR, while it is the most stable one according to hamming-loss standard deviation.

We generated 15 descriptive attributes for odorous molecule data [24] based on the name and formula of molecules. TABLE IV as TABLE III shows that there is no significant difference between using the measure $\mathbb{I}=0$ and the measure $\mathbb{I}=1$ according to hamming-loss. Indeed, by allowing more label relations to be learned $(\mathbb{I}=1)$ more true positive labels are predicted, hence the sensitivity (true positive rate) is increased and hamming-loss is decreased, but due to error propagation, more false positive labels are predicted and consequently hamming-loss is increased. This explains why using $\mathbb{I}=1$ is not always better.

According to the low sensitivity obtained in TABLE IV, the 15 generated attributes are not relevant to discern labels (molecule odours). The prediction error rate in overlapping regions may be high. This problem can be answered by outputting for each data a set of possible labels for each membership grade [25], because experts prefer to have one true prediction in a small set of possible predictions instead of one prediction that could be totally wrong.

\begin{tabular}{|c|l|l|}
\hline meta-classifier & $\begin{array}{l}\text { hamming-loss average } \\
\text { and standard deviation }\end{array}$ & $\begin{array}{l}\text { sensitivity average and } \\
\text { standard deviation }\end{array}$ \\
\hline PSI-MC $(I=1)$ & $0.0350 \pm 0.0155$ & $0.1923 \pm 0.2736$ \\
\hline PSI-MC $(I=0)$ & $0.0306 \pm 0.0128$ & $0.1523 \pm 0.2563$ \\
\hline
\end{tabular}

TABLE IV

THE IMPACT OF CHAINING INTEREST MEASURE IN MOLECULE DATA

Label relation dilemma has also an impact on classifier complexity. TABLE V shows the average number of nodes, leafs, and dependent nodes over all decision trees in each meta- 
classifier, including the initial meta-classifier with cyclic dependencies $H^{0}$. When descriptive attributes are good enough to discern labels (BelaE-5 and BelaE-10), the classifier complexity (number of nodes and leafs) is reduced by learning more label relations (number of dependent nodes), but the opposite happens when descriptive attributes can not discern labels well enough (molecule data), because more dependent labels will be needed and consequently the complexity is increased.

\begin{tabular}{rr|r|r|r|}
\hline Dataset & meta-classifier & node average & leaf average & dependent node average \\
\hline \multirow{3}{*}{ BelaE-5 } & $H^{0}$ & 752 & 376 & 26 \\
& PSI-MC(I=1) & 757 & 379 & 13 \\
& PSI-MC(I=0) & 761 & 381 & 5 \\
BelaE-10 & $H^{0}$ & 748 & 375 & 58 \\
& PSI-MC(I=1) & 761 & 381 & 31 \\
& PSI-MC(I=0) & 772 & 386 & 6 \\
molecules & $H^{0}$ & 115 & 61 & 36 \\
& PSI-MC(I=1) & 95 & 51 & 19 \\
& PSI-MC(I=0) & 46 & 25 & 4 \\
\hline
\end{tabular}

TABLE V

THE IMPACT OF CHAINING INTEREST MEASURE ON CLASSIFIER COMPLEXITY

\section{CONCLUSIONS AND FUTURE WORK}

In this paper, we proposed a new meta-classifier for GMLC named PSI-MC. It is based on three measures named PSImeasures for pre-selection, selection and chaining interest measure. PSI-MC has two main advantages: it allows learning label relations without fixing a predefined relation structure, and it allows controlling the compromise between handling label relations and limiting error propagation. Experiment results on real datasets shows that PSI-MC is competitive with other existing approaches according to hamming-loss, and it is the most stable one according to hamming-loss standard deviation.

In this paper, the impact of PSI-measures is not fully analysed, there is many interesting measures to be studied, and there is even more if we use base classifiers outputting attribute weights such as neural networks. For future work, we plan to study further our proposed classifier, by analysing different PSI-measures, with different base classifiers and for different datasets.

\section{REFERENCES}

[1] W. Cheng, K. Dembczynski, and E. Hllermeier., "Graded multilabel classification: The ordinal case," in Proceedings of LWA2010 - WorkshopWoche: Lernen, Wissen \& Adaptivitaet, M. Atzmller, D. Benz, A. Hotho, and G. Stumme, Eds., Kassel, Germany, 2010.

[2] G. Tsoumakas and I. Katakis, "Multi-label classification: An overview," Int J Data Warehousing and Mining, vol. 2007, pp. 1-13, 2007.

[3] E. A. Cherman, N. Spolaôr, J. Valverde-Rebaza, and M. C. Monard, "Lazy multi-label learning algorithms based on mutuality strategies," Journal of Intelligent \& Robotic Systems, vol. 80, no. 1, pp. 261-276, 2015.

[4] C. Liu and L. Cao, A Coupled k-Nearest Neighbor Algorithm for Multilabel Classification. Cham: Springer International Publishing, 2015, pp. 176-187.
[5] X. Yan, W. Li, Q. Wu, and V. S. Sheng, A Double Weighted Naive Bayes for Multi-label Classification. Singapore: Springer Singapore, 2016, pp. 382-389.

[6] J. Wang, J. Feng, X. Sun, S.-S. Chen, and B. Chen, SimplifiedConstraints Rank-SVM for Multi-label Classification. Berlin, Heidelberg: Springer Berlin Heidelberg, 2014, pp. 229-236.

[7] Z. Sun, Z. Guo, M. Jiang, X. Wang, and C. Liu, Research and Application of Fast Multi-label SVM Classification Algorithm Using Approximate Extreme Points. Cham: Springer International Publishing, 2016, pp. 39-52.

[8] G. Madjarov and D. Gjorgjevikj, Hybrid Decision Tree Architecture Utilizing Local SVMs for Multi-Label Classification. Berlin, Heidelberg: Springer Berlin Heidelberg, 2012, pp. 1-12.

[9] X. Wang, S. An, H. Shi, and Q. Hu, Fuzzy Rough Decision Trees for Multi-label Classification. Cham: Springer International Publishing, 2015, pp. 207-217.

[10] P. M. Ciarelli, E. Oliveira, and E. O. T. Salles, "Multi-label incremental learning applied to web page categorization," Neural Computing and Applications, vol. 24, no. 6, pp. 1403-1419, 2014.

[11] S. Agrawal, J. Agrawal, S. Kaur, and S. Sharma, "A comparative study of fuzzy pso and fuzzy svd-based rbf neural network for multi-label classification," Neural Computing and Applications, pp. 1-12, 2016.

[12] G. Tsoumakas, I. Katakis, and I. Vlahavas, Data Mining and Knowledge Discovery Handbook. Boston, MA: Springer US, 2010, ch. Mining Multi-label Data, pp. 667-685.

[13] J. Read, "A Pruned Problem Transformation Method for Multi-label classification," in Proc. 2008 New Zealand Computer Science Research Student Conference (NZCSRS 2008), 2008, pp. 143-150.

[14] J. Read, B. Pfahringer, G. Holmes, and E. Frank, "Classifier chains for multi-label classification," Mach. Learn., vol. 85, no. 3, pp. 333-359, Dec. 2011.

[15] J. Read, L. Martino, P. M. Olmos, and D. Luengo, "Scalable multi-output label prediction: From classifier chains to classifier trellises," Pattern Recognition, vol. 48, no. 6, pp. 2096 - 2109, 2015.

[16] E. Hüllermeier, J. Fürnkranz, W. Cheng, and K. Brinker, "Label ranking by learning pairwise preferences," Artificial Intelligence, vol. 172, no. 1617, pp. 1897 - 1916, 2008.

[17] J. Fürnkranz, E. Hüllermeier, E. Loza Mencía, and K. Brinker, "Multilabel classification via calibrated label ranking," Machine Learning, vol. 73, no. 2, pp. 133-153, 2008.

[18] L. Zadeh, "Fuzzy sets," Information and Control, vol. 8, no. 3, pp. 338 $-353,1965$.

[19] F. Eibe and H. Mark, "A simple approach to ordinal classification," in Proceedings of the 12th European Conference on Machine Learning, ser. EMCL '01. London, UK, UK: Springer-Verlag, 2001, pp. 145-156.

[20] J. S. Cardoso and J. F. P. da Costa, "Learning to classify ordinal data: the data replication method," Journal of Machine Learning Research, vol. 8, pp. 1393-1429, 2007.

[21] C. Brinker, E. L. Menca, and J. Frnkranz, "Graded multilabel classification by pairwise comparisons." in ICDM, R. Kumar, H. Toivonen, J. Pei, J. Z. Huang, and X. Wu, Eds. IEEE Computer Society, 2014, pp. 731-736.

[22] M. Hall, E. Frank, G. Holmes, B. Pfahringer, P. Reutemann, and I. H. Witten, "The weka data mining software: An update," SIGKDD Explor. Newsl., vol. 11, no. 1, pp. 10-18, Nov. 2009.

[23] J. R. Quinlan, C4.5: Programs for Machine Learning. San Francisco, CA, USA: Morgan Kaufmann Publishers Inc., 1993.

[24] S. Arctander, Perfume and Flavor Chemicals: (aroma Chemicals), ser. Perfume and Flavor Chemicals: Aroma Chemicals. Allured Publishing Corporation, 1969.

[25] K. Laghmari, M. Ramdani, and C. Marsala, "A distributed graph based approach for rough classifications considering dominance relations between overlapping classes," in SITA'15, Intelligent Systems Theories and Applications, 2015 10th Inte. Conf. on, Oct 2015, pp. 1-6. 